\title{
Management of Post Herpetic Neuralgia: Brief Report of Practical Actions Majid Avijgan*
}

Department of Traditional Medicine, Isfahan University of Medical Sciences, Isfahan, Iran

*Corresponding author: Majid Avijgan, Department of Traditional Medicine, Isfahan University of Medical Sciences, P.O.Box 759, Al-Zahra Hospital, Soffe St, Isfahan, Iran. Tel: +46761196285, E-mail: avijgan@yahoo.com

Received date: January 17, 2018; Accepted date: January 23, 2018; Published date: January 29, 2018

Copyright: (c) 2018 Avijgan M. This is an open-access article distributed under the terms of the Creative Commons Attribution License, which permits unrestricted use, distribution, and reproduction in any medium, provided the original author and source are credited.

\begin{abstract}
Post Herpetic Neuralgia (PHN) is one of most common diseases in elderly. It may occur in about $5 \%$ of patients of PHN after acute phase of herpes zoster or zona. PHN has various course with variable and different pain in affected area which some of them may be intractable to be treated. According to previous experiences (during last 20 years), this report will try to present all practical manipulations in regard to PHN pain management.
\end{abstract}

Keywords PHN; Acupuncture; Manipulation; Paradox phenomena

\section{Introduction}

In recent publication we present a complete review on PHN treatment (practically and theory) [1], but this brief report is mainly focused on the practical manipulation of treatment. Based on Traditional Chinese Medicine (TCM) there is three pattern of disharmony for Post Herpetic Neuralgia (PHN): Fire blazing, DampHeat and qi/Blood stagnation which the latter is main cause of pain in $\mathrm{PNH}[1]$.

The pathogenesis of burning pain in PHN is qi/blood stagnation. This is due to obstruction of produced Qi [2] which this latter moves blood. This is called "hypochondriac pain" or "pain in chest" in TCM. This causes a prickling like severe pain in the areas innervated by intercostal areas nerves and presented as an intense and intractable PHN for long time (sometimes several months before visit) and easy to be cured. In clinical experiences there are patients which insomnia for several weeks. This type of PHN is most common clinical and chronic pain.

Based on this classification the determination of pain type, such as allodynia, burning pain, hypoesthesia, and anesthesia, is a critical step for patient's management. The fixed burning pain is due to qi/Blood stagnation which is focus of this clinical round up. Due to for the treatment of various pain several manipulations can be used that are:

\section{Simultaneous needling}

Simultaneous needling (qi ci) technique is for relieving the burning pain as the first step. It is done by "needling in the center of a pain area or lesion and then also needling to right and left of central point" [3]. This protocol is effective along with Electero acupuncture (EAP) [4] and based on my experience even without it [1]. The muscles and flesh should be twitched by electro-stimulation $[1,4]$. They can be used as local points.

\section{Huatuojiaji points acupuncture}

But as the distance Acupuncture Points (AP), the Huatuojiaji points at the vertebral level corresponding to the location of the herpes zoster for treatment of PHN produce an obvious analgesic effect $[1,5]$. This method is more effective in new cases than chronic cases [6]. It seems that they may be effective by analgesic dermatomal effect which is act better in acute cases.

\section{Surrounding needling}

The chronic pain resulted from stagnation can be treated by Qi and blood movement by method of surrounding needling, or "Surround the dragon". This method can also be accompanied with or without Electro Acupuncture) [7]. Horizontally puncturing along the innervating areas of PHN gives rise for dispersing stasis of blood and removing obstruction of collateral [2]. Needles, around the lesions without regard to traditional acupuncture points, are inserted at $2 \mathrm{~cm}$ intervals subcutaneously at a 25 degree angle to the skin around the rash of PHN. No deqi, or energy sensation need to be obtained. The number of needles depends entirely on the extensiveness of the fixed painful area [1].

\section{Plum blossom}

Due to chronic qi and blood stasis there is sometimes telangectasia in area of PHN [1]. In these cases the invasive method such as plum blossom has more analgesic effect than other therapies. This method can be applied even in the case of acute herpes zoster too [8].

\section{Cupping}

Additionally, in some chronic cases cupping can help to remove the qi and blood stagnation. The stagnated produced qi [2] and blood in the collaterals can be reduced by moving capping and or fixed cupping after plum blossom [1].

After plum blossom needling the cups were placed over the area to encourage bleeding from the local area which will disappear the stagnation and also telangectasia [1].

\section{Paradox phenomen}

In our patients a new clinical phenomen was observed in nearly $\% 100$ of PHN due to Blood/qi stagnation type. This new finding, that in the best of my knowledge is the first time to be suggested, is Paradox Phenomen (It may be called Avijgan Phenomen). 
Citation: Avijgan M (2018) Management of Post Herpetic Neuralgia: Brief Report of Practical Actions. Altern Integr Med 7: 259. doi: 10.4172/2327-5162.1000259

Page 2 of 2

In some cases the day after the first moving cupping there is a worsening of pain which lead to even more complaint. In this case, should notice the patients that pain to be probably worsened and then patients will be ready to accept it. This kind of pain will be disappeared within one to two days and not any contraindication for further cupping [1].

\section{Conclusion}

PHN due to qi/blood stagnation type is a chronic burning pain (sometime several months and even years) with lead to disability of suffered patients. By this step by step (in order of number) approach the nearly complete pains relief can be achieved [1].

\section{References}

1. Majid A, Hajzargarbashi ST, Kamran A, Mahtab A (2017) Post herpetic Neuralgia: Practical Experiences: Return to Traditional Chinese Medicine. J Acupunct Meridian Stud 10: 157-164.
2. Avijgan M (2013) Can the Primo Vascular system (Bong Han Duct) be a Basic Concept for Qi Production? Int J Integr Med 1: 20.

3. Wang MQ, Yu SF (1987) Herpes zoster treated by AP: Report of 50 cases. Beijing JTCM 2: 37-38.

4. Jie H (2005) Simultaneous Needling Plus Electro-acupuncture Treatment of 40 Cases of Geriatric Post-herpetic Neuralgia." Zhongguo Zhen Jiu $1: 48$.

5. Serres G (1988) Comments on the technique of the treatment of herpes zoster. Acupunct Res 13: 7-9.

6. Tanabe S, Shiba K (1984) The effect of AP for herpetic pain. J Jpn Soc Acupunct 33: 383-387.

7. Shi Y (1993) AP treatment for 5 cases of herpes zoster accompanying AIDS. Shanghai J Acupunct Moxibust 12:119.

8. Chen JX, Feng SH (1984) Treatment of herpes zoster by plum-blossom needling: A clinical observation of 110 cases. J New Chin Med 7:29. 